\title{
Exposure to odours improves olfactory function in healthy children*
}

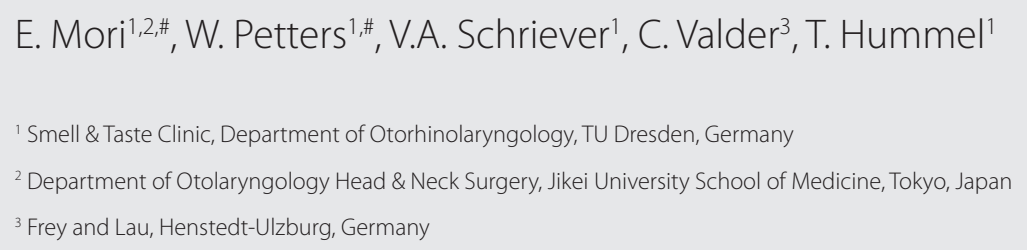

\author{
Rhinology 53: 221-226, 2015 \\ DOl:10.4193/Rhino 14.192 \\ *Received for publication: \\ July 14, 2014
}

Accepted: January 8, 2015

" Both authors contributed equally

\begin{abstract}
Background: Short-term exposure to odours, also called "olfactory training" has been shown to improve olfactory function in healthy people but also in people with olfactory loss. Aim of this single center, prospective, controlled study was to investigate the change of olfactory function following twice-daily, short-term exposure to 4 odours over a period of approximately 12 weeks.
\end{abstract}

Material and Methods: We compared odour identification abilities and odour thresholds between an olfactory training group (TR group) and a group that did not perform such training (noTR group). Participants exposed themselves twice daily to 4 odours ("rose", "eucalyptus", "lemon", "clove"). Olfactory testing was performed before and after the training period using the "Sniffin' Sticks" test kit (odour identification plus odour thresholds).

Results: At baseline the two groups were not significantly different in terms of age and measures of olfactory sensitivity. The TR group performed significantly better for odour thresholds for all 4 odours compared to the noTR group after 12 weeks of olfactory training. Also, with regard to odour identification the TR group outperformed the noTR group. No significant differences were found for diary-based intensity ratings.

Conclusion: Repeated exposure to odours seems to improve general olfactory sensitivity in children.

Key words: olfaction, anosmia, smell, regeneration

\section{Introduction}

Short-term exposure to odours, also called "olfactory training" has been shown to improve olfactory function in healthy people but also in people with olfactory loss ${ }^{(1-4)}$. Older people responded to olfactory training only tentatively ${ }^{(5)}$. This indicated that the olfactory system may become less plastic in relation to age. If this was true, it could be concluded that olfactory training would work best in children.

Aim of this single center, prospective, controlled study was to investigate the change of olfactory function following twice-daily, short-term exposure to 4 odours over a period of approximately 12 weeks. We compared odour identification abilities and odour thresholds between an olfactory training group and a group that did not perform such training.

\section{Materials and methods}

\section{Participants}

All participants and their parents/legal representatives provided written informed consent. The experimental design was approved by the Ethics Committee of the Medical Faculty of the TU Dresden (study number EK386112011). 
A total of 72 children participated in the study ( 41 girls, 31 boys). Based on previous studies (e.g. ${ }^{(6,7)}$ ) this sample size appeared to be adequate to study potential effects of the training procedure. The mean age was 11.6 years (range 9-15 years). The participants received a thorough, standardized history related to potential causes of olfactory loss. Exclusion criteria were chronic sinunasal disease, or acute nasal diseases, e.g., acute viral infections or acute allergies. In the training group (TR) were 29 girls and 11 boys (mean age 11.5 years, range 9-15 years), in the nontraining group (noTR) were 12 girls and 20 boys, respectively (mean age 11.7 years, range 9-14 years). We did not calculate a sample size because experience on olfactory training sofar is only available on adults; in addition, the study was thought to be absolutely harmless so that there was no reason to calculate the lowest possible number of subjects that should be included in this study. According to our experience, however, a size of 30-40 individuals per group appeared to be sufficient to demonstrate effects of olfactory training on the sense of smell.

Although the composition of both groups was similar in terms of age and pre-existing olfactory function, they differed in age (Fisher's exact test: $p=0.0041$ ). Although there was no randomization plan, subjects were included in the training or no-training group based on the sequence how they were presented them to the experimenter.

\section{Training with odorants}

Olfactory training was performed over a period of 12 weeks. Participants exposed themselves twice daily to 4 odours ("rose", "eucalyptus", "lemon", "clove"). Different from previous studies, however, we did not use single molecules (e.g., phenyl ethyl alcohol [PEA], eucalyptol, citronellal, eugenol) but complex mixtures of odours that were especially prepared for the use in children (odours provided by Frey and Lau, Henstedt-Ulzburg, Germany; rose: order number P0604034; lemon: P0119551; eucalyptus: P0113975; clove: P0114500). This was done to not expose the participants to any even remotely possible toxic concentrations of odorants used. The 4 odours were chosen to be representative of 4 odour categories claimed by Henning ${ }^{(8)}$ in his work on the "odour prism" ("Geruchsprisma") where he tried to identify primary odours (compare $\left.{ }^{(9)}\right)$. The categories are flowery ["blumig;" e.g., rose], foul ["faulig"], fruity ["fruchtig;" e.g., lemon], aromatic ["würzig;" e.g., clove], burnt ["brenzlich"], and resinous ["harzig;" e.g., eucalyptus]. For training participants received 4 brown glass jars (total volume $50 \mathrm{ml}$ ) with the 4 odours in it ( $4 \mathrm{ml}$ each, soaked in cotton pads to prevent spilling). All jars were labelled with the odour name.

Participants in the TR group were advised to sniff the odours in the morning and in the evening for approximately 10 seconds each. To additionally focus the attention on the training, they were asked to keep a "diary" where they rated the intensity of each of the four odours each Sunday; ratings ranged between 0 (no odour perceived) and 10 (very strong odour). Participants in the noTR group received no such instructions.

Compliance with training was not explicitly tested, although children of the TR group maintained upon questioning that they had performed the training. Parents also confirmed these statements.

\section{Olfactory testing}

Olfactory testing was performed before and after the training period of 12 weeks using the "Sniffin' Sticks" test kit ${ }^{(10)}$, which involved tests for odour threshold, and odour identification (Burghart, Wedel, Germany). Using commercially available felt-tip pens, the odorants were presented approximately 2 $\mathrm{cm}$ in front of both nostrils for 2 seconds. PEA odour threshold was assessed by a single-staircase, 3-alternative forced choice (3-AFC) procedure in blindfolded subjects. Three pens were presented to the participant in a randomized order, two contained odorless solvent and the other odorant in a certain dilution. The participants' task was to indicate the pen with the odorant. Concentration was increased if one of the blanks was chosen, and it was decreased if the correct pen was identified twice in a row. The mean of the last 4 of a total of 7 reversal points was used as detection threshold (ranging from 1 to 16 ). A total of 16 odour concentrations were tested starting from a $4 \%$ stock solution (dilution ratio 1:2; solvent propylene glycol).

For testing of odour identification 16 pens containing common odours were offered. The participant had to identify each of the odorants from a list of four descriptors. For 16-item odour identification testing with the Sniffin' Sticks in children aged 5-15 years scores indicating hyposmia are 10 and less; for odour thresholds these scores are 4.50 or less ${ }^{(11)}$. According to a study in adults clinical improvement is seen at scores of 5.75 and higher ${ }^{(12)}$.

\section{Threshold measures}

While thresholds for PEA were measured using the single staircase paradigm within the "Sniffin' Sticks" test kit (see above), thresholds for the other odours used for training (eucalyptus, clove, lemon) were assessed by means of the method of ascending limits (e.g., $\left.{ }^{(13)}\right)$, using a 3-AFC procedure. This procedure was chosen because it is slightly faster than the staircase procedure although it may be less reliable ${ }^{(14)}$.

Odours were presented in brown glass jars using a procedure similar to the presentation of PEA with the "Sniffin' Sticks". Two of the jars contained odorless solvent (propylene glycol; Sigma, Deisenhofen, Germany), the other one odorant in a certain concentration. The participants' task was to indicate the jar with the odorant. Correct identification was assumed when the participant correctly identified the same odour concentration 3 times in a row. A total of 8 concentrations for each odour were tested 

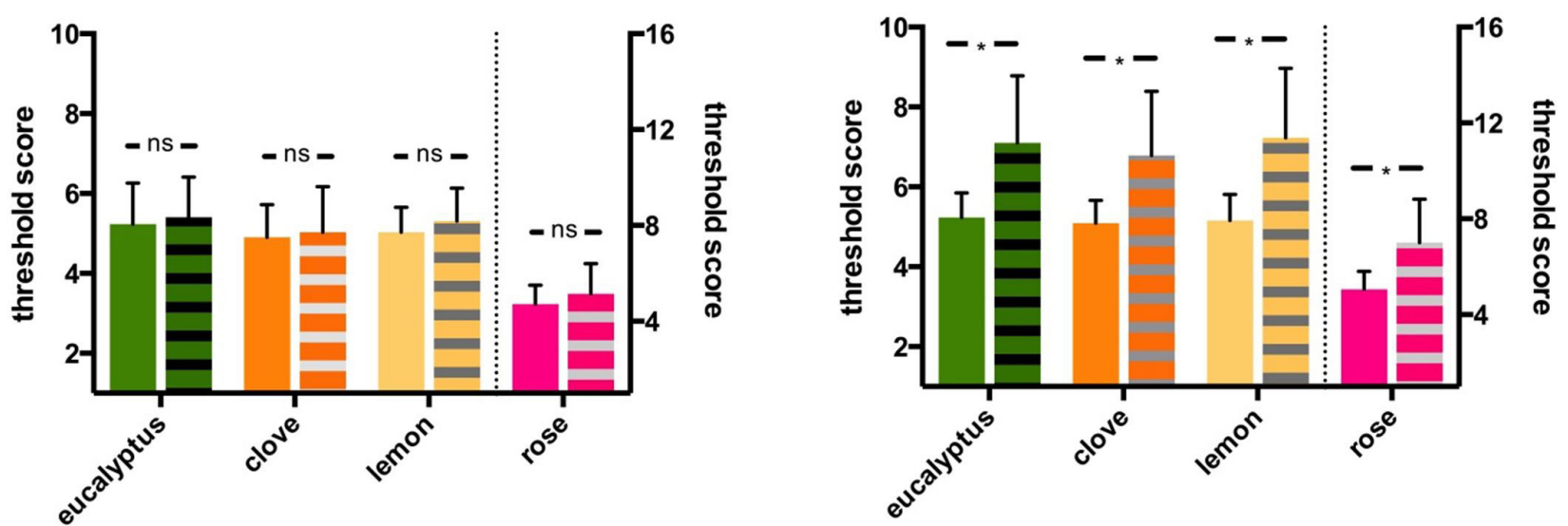

Figure 1. The graphs show mean ( \pm standard errors) results for odour thresholds children who did (right) and who did not (left) perform olfactory training, separately for odour thresholds obtained before (solid bars) and after (bars with stripes) the intervention. The left hand scale relates to thresholds obtained for the odours eucalyptus, cloves, and lemon, the right hand scale relates to thresholds measured for rose odour, because odours were resented slightly differently - rose in pens, the other odours in bottles. Higher scores indicate higher sensitivity. Significant differences between measures before and after are indicated with an asterisk.

starting from $4 \%$ stock solutions (dilution ratio 1:4; solvent propylene glycol). Between tests of the odorants subjects rested for approximately $5 \mathrm{~min}$ to minimize desensitization.

\section{Statistical analysis}

For statistical analyses, SPSS (Statistical Packages for Social Sciences, version 21.0, SPSS Inc., Chicago, IL, USA) was used. Comparisons between the two groups were performed using t-tests for independent samples and $\mathrm{Chi}^{2}$ tests. Correlation analyses were performed according to Pearson. The alpha-level was set at 0.05 .

\section{Results}

At baseline the two groups were not significantly different in terms of age and measures of olfactory sensitivity (Table 1) (age: $\mathrm{t}=0.60, \mathrm{p}=0.55$; odour identification: $\mathrm{t}=1.87, \mathrm{p}=0.07$; odour thresholds: all t's $<1.64$, all p's $>0.11$ ).

When using t-tests for comparison of differences between thresholds obtained after minus those obtained before training, the TR group performed significantly better for all 4 odours compared to the noTR group (all ts $>4.51$, all ps $<0.001$ ) (Table 1; Figure 1). With regard to odour identification, on average, participants in the TR group outperformed the noTR group although this was not significant (mean difference between score after minus score before training: TR: $M=1.25, S D=1.79$; noTR: $M=0.56, S D=1.48 ; t=1.74, p=0.09$ ). However, when disregarding those pens in the odour identification task the odours of which had been used for training, TR group subjects significantly outperformed noTR group subjects (mean difference between score after minus score before training: TR: $M=1.28, S D=1.57$; noTR: $M=0.13, S D=2.53 ; t=2.36, p=0.021$ ).

Evaluation of the "smell diary" showed no changes for odor intensity ratings made during the course of the training (MANOVA for repeated measures separately for the 4 trained odours: all ps $>0.15$ ); in addition comparisons of intensity ratings obtained before training and those 12 weeks after training did not yield any significant differences (all ps $>0.50$ ).

\section{Discussion}

The present results indicate that olfactory training improves olfactory sensitivity in children and adolescents at the level of odour thresholds and at the level of odour identification. At the level of ratings no such effect is seen.

As a rare exception in the central nervous system olfactory receptor neurons (ORN) constantly turn over ${ }^{(15-17)}$. In addition, mitral/tufted cells exhibit continuous synaptogenesis in the olfactory bulb ${ }^{(18)}$. Exposure to odour seems to affect these regenerative processes ${ }^{(19)}$. According to this, repeated exposure to odours (termed "olfactory training") has been reported to improve olfactory function in patients with olfactory loss ${ }^{(1-3)}$. In addition, as a morphological expression of this improvement the volume of the olfactory bulb has been found to increase after such an olfactory training ${ }^{(20,21)}$. However, exposure to odours may also improve the general interest in odours so that they might have a higher motivation to perform well in the odour task, although such an effect does not explain the increase in olfactory bulb volume seen in other studies ${ }^{(20,21)}$. 
Table 1. Means and standard deviations (SD) of results obtained at baseline and after the training period separately for the 2 groups (no training $[\mathrm{n}=$ $32]$ / training $[n=40]$ ). The Table contains also the results of the statistical comparison between groups using t-tests for independent samples (results obtained after training minus results obtained at baseline). The variable "identification 16 item" shows results for the classical 16-item Sniffin Sticks test; the available "identification 13-item" shows results without scores for the odours of "rose", "lemon" and "cloves" (see text for details).

\begin{tabular}{|c|c|c|c|c|c|c|c|}
\hline & & \multicolumn{2}{|c|}{ notR group ( $n=32$ ) } & \multicolumn{2}{|c|}{ TR group $(n=40)$} & \multicolumn{2}{|c|}{ t-test - between groups } \\
\hline & & mean & SD & mean & SD & t-value & p-value \\
\hline eucalyptus & baseline & 5.23 & 1.03 & 5.41 & 1.00 & 4.52 & $<0.001$ \\
\hline threshold & end & 5.25 & 0.62 & 7.10 & 1.68 & & \\
\hline clove & baseline & 4.90 & 0.82 & 5.03 & 1.14 & 5.02 & $<0.001$ \\
\hline threshold & end & 5.09 & 0.57 & 6.79 & 1.60 & & \\
\hline rose & baseline & 4.72 & 0.79 & 5.14 & 1.27 & 4.68 & $<0.001$ \\
\hline threshold & end & 5.06 & 0.74 & 7.01 & 1.81 & & \\
\hline lemon & baseline & 5.03 & 0.62 & 5.31 & 0.82 & 5.92 & $<0.001$ \\
\hline threshold & end & 5.16 & 0.65 & 7.23 & 1.74 & & \\
\hline identification & baseline & 12.34 & 1.68 & 11.60 & 1.68 & 1.60 & 0.11 \\
\hline 16 item & end & 12.91 & 1.25 & 12.85 & 1.48 & & \\
\hline identification & baseline & 10.16 & 1.30 & 9.40 & 1.55 & 2.20 & 0.03 \\
\hline 13 item & end & 10.28 & 2.10 & 10.68 & 1.27 & & \\
\hline
\end{tabular}

On the other hand, in animals neurogenic activity of the olfactory nerve is more efficient in younger than in adult individuals ${ }^{(22)}$. Compared to adults, olfactory dysfunction in children seems to resolve faster and more effectively for example after bone marrow transplantation ${ }^{(23)}$. In addition, the olfactory bulb volume in children has been shown to correlate with olfactory function ${ }^{(24)}$. Based on this it may be assumed that children may benefit more strongly from olfactory training than adults. This is indicated by the present data in comparison to previous results in older adults where olfactory training exhibited only a slight effect on odour thresholds, but no effect on odour identification ${ }^{(5)}$. Although olfactory training may still have its place in older adults with the possibility of preventing loss of olfactory function, probably based on higher plasticity children seem to outperform older adults.

It is important to note that participants in this study not only improved in sensitivity for the trained odours but that their seemed to be a general improvement of olfactory sensitivity as indicated by the increase in odour identification scores. Similar effects have also been observed for olfactory training in adults (e.g., ${ }^{(4,25)}$ ). However, in this group it was especially interesting to see that the improvement in general odour identification came out best when the performance on the trained odours was disregarded. The likely explanation for this discrepancy lies in the fact that participants trained with certain mixtures of odours, resembling very nicely the complex smells of rose, clove, eucalyptus, and lemon. In the test with the "Sniffin' Sticks", however, single molecules are used to resemble the smell of rose (phenyl ethyl alcohol), clove (eugenol), and lemon (citronellal). It can be assumed that the children became experts on the complex odours they trained with. When then confronted with the single molecules they no longer recognized them as rose etc., but searched for alternative, different descriptors.

With regard to thresholds, it should be noted that a more complete assessment of thresholds would have been with the inclusion of a novel odour instead of the rose concept.

When studying the ratings made in the diaries no significant increase of the intensity ratings was found over time. In light of the clear changes of measured olfactory function this indicates that ratings can be problematic, for various reasons, one of them being the tendency of subjects to provide stable responses. In the present design of the diaries subjects always were aware of their rating history which may have affected their actual ratings. Another aspect could be that ratings of olfactory abilities in ge- 
neral do not correlate very well with measures of olfactory function (26-28).

As olfactory function seems to be improved in children after training, it may be speculated how this might affect the lives of these individuals if an interest in odours would be a lasting change ${ }^{(29)}$. It may be speculated that it might have an effect on the personality of children as people with higher olfactory sensitivity have been shown to seek more compromise than people who are less sensitive ${ }^{(30)}$. It may also be that children with a higher olfactory sensitivity enjoy their foods and drinks to a higher degree which may have consequences for their diets ${ }^{(31,32)}$.

One concern about the results may relate to the gender distribution with the training group performed better and had significantly more girls. At this age (11 years mean) girls tend to be a little in advance with cognitive functions and girls/women outperform men in olfactory tasks (robust effect along many studies). Furthermore, a previous study by Dalton et al. ${ }^{(1)}$ showed that there is a gender specific olfactory learning for certain odours in favor of female gender. In light of these facts it becomes very difficult, although the study is interesting to waive away doubts about gender and thus selection bias. It is a pity that this prospective study did not select in a randomized way; this would probably have avoided that error. Is the improvement a gender or training effect? However, when comparing only girls then the effects for odour thresholds are also highly significant (for all 4 trained odours p's are $<0.001$ ). The same is true when comparing boys only (all p's $<0.014)$. In conclusion, repeated exposure to odours seems to improve general olfactory sensitivity in children.

\section{Author contributions}

All authors contributed to the conception and the conduction of the study; all authors contributed to the analysis and interpretation of the results, and all authors contributed to the final manuscript.

\section{Conflict of interest}

The authors declare there are no conflicts of interest.

\section{References}

1. Hummel T, Rissom K, Hähner A, Reden J, Weidenbecher M, Hüttenbrink KB. Effects of olfactory training in patients with olfactory loss. Laryngoscope. 2009; 119: 496-499.

2. Damm M, Pikart LK, Reimann $\mathrm{H}$, et al. Olfactory training is helpful in postinfectious olfactory loss - a randomized controlled multicenter study. Laryngoscope. 2014; 124: 826-831.

3. Haehner A, Tosch C, Wolz M, et al. Olfactory training in patients with Parkinson's disease. PLoS One. 2013; 8: e61680.

4. Konstantinidis I, Tsakiropoulou E, Bekiaridou P, Kazantzidou C, Constantinidis J. Use of olfactory training in post-traumatic and postinfectious olfactory dysfunction. Laryngoscope. 2013; 123: E85-90.

5. Schriever VA, Lehmann S, Prange J, Hummel T. Preventing olfactory deterioration: olfactory training may be of help in older people. J Am Geriatr Soc. 2014; 62: 384-386.

6. Hummel T, Heilmann S, Huttenbriuk KB. Lipoic acid in the treatment of smell dysfunction following viral infection of the upper respiratory tract. Laryngoscope. 2002; 112: 2076-2080.

7. Quint C, Temmel AFP, Hummel T, Ehrenberger $\mathrm{K}$. The quinoxaline derivative caroverine in the treatment of sensorineural smell disorders: a proof of concept study. Acta Otolaryngol. 2002; 122: 877-881.

8. Henning H. Der Geruch. Leipzig: Johann Ambrosius Barth, 1916.

9. Amoore JE. Specific anosmia and the concept of primary odors. Chem Sens Flav. 1977; 2: 267-281.

10. Hummel T, Sekinger B, Wolf SR, Pauli E, Kobal G. 'Sniffin' sticks': olfactory perfor- mance assessed by the combined testing of odor identification, odor discrimination and olfactory threshold. Chem Senses. 1997; 22: 39-52.

11. Hummel T, Kobal G, Gudziol H, Mackay-Sim A. Normative data for the "Sniffin' Sticks" including tests of odor identification, odor discrimination, and olfactory thresholds: an upgrade based on a group of more than 3,000 subjects. Eur Arch Otorhinolaryngol. 2007; 264: 237-243.

12. Gudziol V, Lotsch J, Hahner A, Zahnert T, Hummel T. Clinical significance of results from olfactory testing. Laryngoscope. 2006; 116: 1858-1863.

13. Cain WS, Gent JF, Goodspeed RB, Leonard G. Evaluation of olfactory dysfunction in the Connecticut Chemosensory Clinical Research Center (CCCRC). Laryngoscope. 1988; 98: 83-88.

14. Doty RL, McKeown DA, Lee WW, Shaman P. A study of the test-retest reliability of ten olfactory tests. Chem Senses. 1995; 20: 645-656.

15. Calof AL, Hagiwara N, Holcomb JD, Mumm JS, Shou J. Neurogenesis and cell death in olfactory epithelium. J Neurobiol. 1996; 30: 67-81.

16. Calof AL, Mumm JS, Rim PC, Shou J. The neuronal stem cell of the olfactory epithelium. J Neurobiol. 1998; 36: 190-205.

17. Graziadei PPC, Monti-Graziadei GA Continuous nerve cell renewal in the olfactory system. In: Jacobson M, ed. Handbook of sensory physiology, vol. IX. New York: Springer, 1978: 55

18. Lledo PM, Gheusi G. Olfactory processing in a changing brain. Neuroreport. 2003; 14: 1655-1663.
19. Wang L, Chen L, Jacob T. Evidence for peripheral plasticity in human odour response. J Physiol. 2004; 554: 236-244.

20. Haehner A, Rodewald A, Gerber JC, Hummel T. Changes of the volume of the human olfactory bulb with olfactory function. Arch ORL. 2008; 134: 621-624.

21. Gudziol V, Buschhuter D, Abolmaali N, Gerber J, Rombaux P, Hummel T. Increasing olfactory bulb volume due to treatment of chronic rhinosinusitis--a longitudinal study. Brain. 2009; 132: 3096-3101.

22. Ducray A, Bondier JR, Michel G, Bon K, Propper A, Kastner A. ecovery following peripheral destruction of olfactory neurons in young and adult mice. Eur J Neurosci. 2002; 15: 1907-1917.

23. Cohen J, Laing DG, Wilkes FJ. Taste and smell function in pediatric blood and marrow transplant patients. Supportive Care Cancer, 2012; 20: 3019-3023.

24. Hummel T, Smitka M, Puschmann S, Gerber JC, Schaal B, Buschhuter D. Correlation between olfactory bulb volume and olfactory function in children and adolescents. Exp Brain Res. 2011; 214: 285-291.

25. Fleiner F, Lau L, Goktas O. Active olfactory training for the treatment of smelling disorders. Ear Nose Throat J. 2012; 91: 198-203, 215.

26. Landis BN, Hummel T, Hugentobler M, Giger R, Lacroix JS. Ratings of overall olfactory function. Chem Senses. 2003; 28: 691-694.

27. Shu CH, Hummel T, Lee PL, Chiu CH, Lin SH, Yuan BC. The proportion of self-rated olfactory dysfunction does not change across the life span. Am J Rhinol Allergy. 2009; 23: 413-416.

28. Nguyen DT, Nguyen-Thi PL, Jankowski R. 
How does measured olfactory function correlate with self-ratings of the sense of smell in patients with nasal polyposis? Laryngoscope. 2012; 122: 947-952.

29. Croy I, Buschhuter D, Seo HS, Negoias S, Hummel T. Individual significance of olfaction: development of a questionnaire. Eur Arch Otorhinolaryngol. 2010; 267: 67-71.

30. Croy I, Springborn M, Lotsch J, Johnston AN, Hummel T. Agreeable smellers and sensitive neurotics--correlations among personality traits and sensory thresholds. PLoS One. 2011; 6: e18701.

31. Coulthard $H$, Blissett J. Fruit and vegetable consumption in children and their mothers. Moderating effects of child sensory sensitivity. Appetite. 2009; 52: 410-415.
32. Aschenbrenner $K$, Scholze N, Joraschky $P$ Hummel T. Gustatory and olfactory sensitivity in patients with anorexia and bulimia in the course of treatment. J Psychiatr Res. 2008; 43: 129-137.
Thomas Hummel, M.D.

Smell \& Taste Clinic

Department of Otorhinolaryngology

TU Dresden

Fetscherstrasse 74

01307 Dresden

\section{Germany}

Tel: +49-351-458-4189

Fax: +49-351-458-4326

E-mail: thummel@mail.zih.tu-dresden.de 